\title{
New Regular Solutions with Axial Symmetry in Einstein-Yang-Mills Theory
}

\author{
Rustam Ibadov \\ Department of Theoretical Physics and Computer Science, \\ Samarkand State University, Samarkand, Usbekistan \\ and \\ Burkhard Kleihaus, Jutta Kunz and Yasha Shnir \\ Institut für Physik, Universität Oldenburg, Postfach 2503 \\ D-26111 Oldenburg, Germany
}

November 11, 2018

\begin{abstract}
We construct new regular solutions in Einstein-Yang-Mills theory. They are static, axially symmetric and asymptotically flat. They are characterized by a pair of integers $(k, n)$, where $k$ is related to the polar angle and $n$ to the azimuthal angle. The known spherically and axially symmetric EYM solutions have $k=1$. For $k>1$ new solutions arise, which form two branches. They exist above a minimal value of $n$, that increases with $k$. The solutions on the lower mass branch are related to certain solutions of Einstein-Yang-Mills-Higgs theory, where the nodes of the Higgs field form rings.
\end{abstract}




\section{Introduction}

The well-known regular Bartnik-McKinnon (BM) solutions [1] and the corresponding non-Abelian black hole solutions [2, are asymptotically flat, static spherically symmetric solutions of SU(2) Einstein-Yang-Mills (EYM) theory. They are unstable solutions, sphalerons [3], and are characterized by the number of nodes of the gauge field. Besides the BM solutions there are also asymptotically flat, static, only axially symmetric regular and black hole solutions [4. These are characterized by two integers, the node number of their gauge field function(s), and the winding number with respect to the azimuthal angle, denoted $n$. The spherically symmetric solutions have winding number $n=1$, while the axially symmetric solutions have winding number $n>1$.

In SU(2) Einstein-Yang-Mills-Higgs (EYMH) theory, with a triplet Higgs field, gravitating monopole solutions arise [5]. While gravitating monopoles with unit magnetic charge are spherically symmetric, the known gravitating multimonopoles possess axial symmetry [6]. As in EYM theory, these EYMH solutions are characterized by two integers, the node number of the gauge field and the azimuthal winding number $n$, which corresponds to the topological charge of the monopoles.

But EYMH theory allows for further static axially symmetric solutions, representing gravitating monopole-antimonopole pair, chain and vortex solutions [7, 8, 9. These solutions can be characterized by the azimuthal winding number $n$, and by a second integer $m$, related to the polar angle. For the monopole-antimonopole chains, which in flat space arise for $n=1$ and 2 , the integer $m$ corresponds to the number of nodes of the Higgs field (and thus the number of poles on the symmetry axis) [8]. In vortex solutions, on the other hand, which in flat space arise for winding number $n>2$, the Higgs field vanishes (for even $m$ ) on $m / 2$ rings centered around the symmetry axis 8.

The existence of monopole-antimonopole pair, chain and vortex solutions in EYMH theory [7, 8, 9] immediately leads to the question, whether there might be analogous solutions in EYM theory, even though there would be no Higgs field participating in the subtle interplay of attraction and repulsion.

In this letter we report the existence of one such new type of solution, related to vortex solutions in EYMH theory.

In section II we present the EYM action, the axially symmetric ansatz and the boundary conditions. In section III we discuss the properties of the 
new axially symmetric solutions, and we present our conclusions in section IV.

\section{Action and Ansatz}

We consider the SU(2) EYM action

$$
S=\int\left(\frac{R}{16 \pi G}-\frac{1}{2} \operatorname{Tr}\left(F_{\mu \nu} F^{\mu \nu}\right)\right) \sqrt{-g} d^{4} x
$$

with Ricci scalar $R$, field strength tensor

$$
F_{\mu \nu}=\partial_{\mu} A_{\nu}-\partial_{\nu} A_{\mu}+i e\left[A_{\mu}, A_{\nu}\right]
$$

gauge potential $A_{\mu}=\tau^{a} A_{\mu}^{a} / 2$, and gravitational and Yang-Mills coupling constants $G$ and $e$, respectively. Variation of the action (III) with respect to the metric $g^{\mu \nu}$ leads to the Einstein equations, variation with respect to the gauge potential $A_{\mu}$ to the gauge field equations.

In isotropic coordinates the static axially symmetric metric reads 4]

$$
d s^{2}=-f d t^{2}+\frac{m}{f} d r^{2}+\frac{m r^{2}}{f} d \theta^{2}+\frac{l r^{2} \sin ^{2} \theta}{f} d \varphi^{2},
$$

where the metric functions $f, m$ and $l$ are functions of the coordinates $r$ and $\theta$, only. The $z$-axis $(\theta=0, \pi)$ represents the symmetry axis. Regularity on the $z$-axis requires $m=l$ there.

For the gauge field we employ the ansatz [4, 8, 9]

$$
A_{\mu} d x^{\mu}=\frac{1}{2 e r}\left[\tau_{\varphi}^{n}\left(H_{1} d r+\left(1-H_{2}\right) r d \theta\right)-n\left(\tau_{r}^{n, k} H_{3}+\tau_{\theta}^{n, k} H_{4}\right) r \sin \theta d \phi\right]
$$

Here the symbols $\tau_{r}^{n, k}, \tau_{\theta}^{n, k}$ and $\tau_{\varphi}^{n}$ denote the dot products of the cartesian vector of Pauli matrices, $\vec{\tau}=\left(\tau_{x}, \tau_{y}, \tau_{z}\right)$, with the spatial unit vectors

$$
\begin{aligned}
\vec{e}_{r}^{n, k} & =(\sin k \theta \cos n \varphi, \sin k \theta \sin n \varphi, \cos k \theta), \\
\vec{e}_{\theta}^{n, k} & =(\cos k \theta \cos n \varphi, \cos k \theta \sin n \varphi,-\sin k \theta), \\
\vec{e}_{\varphi}^{n} & =(-\sin n \varphi, \cos n \varphi, 0),
\end{aligned}
$$

respectively. The gauge field functions $H_{i}, i=1-4$, depend on the coordinates $r$ and $\theta$, only. For $k=n=1$ and $H_{1}=H_{3}=0, H_{2}=1-H_{4}=w(r)$ 
the BM solutions [1] are recovered, while for $k=1, n>1$, one obtains the axially symmetric solutions of 4. The new solutions reported here are obtained for $k>1$. They are related to EYMH solutions with $m=2 k$ in the limit of vanishing Higgs field [9].

The ansatz is form-invariant under the abelian gauge transformation [4]

$$
U=\exp \left(\frac{i}{2} \tau_{\phi}^{n} \Gamma(r, \theta)\right)
$$

We fix the gauge by choosing the gauge condition [4, 8, 9]

$$
r \partial_{r} H_{1}-\partial_{\theta} H_{2}=0
$$

To obtain asymptotically flat solutions which are globally regular and possess the proper symmetries, we need to impose appropriate boundary conditions 4, , 8, 9]. At the origin we impose the boundary conditions

$$
\partial_{r} f=\partial_{r} m=\partial_{r} l=0, \quad H_{1}=H_{3}=H_{4}=0, H_{2}=1,
$$

at infinity we impose

$$
f=m=l=1, \quad H_{1}=H_{3}=0, H_{2}=1-2 k, H_{4}=2 \sin (k \theta) / \sin \theta,
$$

and on the $z$-axis we impose

$$
\partial_{\theta} f=\partial_{\theta} m=\partial_{\theta} l=0, \quad H_{1}=H_{3}=0, \partial_{\theta} H_{2}=\partial_{\theta} H_{4}=0 .
$$

We further introduce the dimensionless coordinate $x$, and the dimensionless mass $\mu$,

$$
x=\frac{e}{\sqrt{4 \pi G}} r, \quad \mu=\frac{e G}{\sqrt{4 \pi G}} M .
$$

\section{Numerical Results}

Subject to the above boundary conditions, we solve the system of seven coupled non-linear partial differential equations numerically. To map spatial infinity to the finite value $\bar{x}=1$, we employ the radial coordinate [4, ㅇ, 9 ]

$$
\bar{x}=\frac{x}{1+x} .
$$


The numerical calculations are based on the Newton-Raphson method, and are performed with help of the program FIDISOL [10. The equations are discretized on a non-equidistant grid in $\bar{x}$ and $\theta$. Typical grids used have sizes $70 \times 30$, covering the integration region $0 \leq \bar{x} \leq 1$ and $0 \leq \theta \leq \pi / 2$. For the method, it is essential to have a good first guess, to start the iteration procedure. For the $k=1 \mathrm{EYM}$ solutions, the $n=1 \mathrm{BM}$ solutions serve as a first guess, and then the 'parameter' $n$ is varied (via unphysical noninteger values) to obtain solutions with higher winding number $n$ [4]. For the $k=2$ solutions we employ the $m=4, n=4 \mathrm{EYMH}$ vortex solution with (almost) vanishing Higgs field as a first guess [9], and then again vary $n$. Similarly, for the $k=3$ solutions we start from the $m=6, n=6 \mathrm{EYMH}$ vortex solution.

In EYMH theory, the monopole-antimonopole pair (MAP) solution is obtained, when $m=2 k=2, n=1$. Here a monopole and an antimonopole are located symmetrically on the $z$-axis. Similarly, one might try to find a sphaleron-antisphaleron pair (SAP) solution in EYM theory. The boundary conditions of such a SAP solution, however, do not differ from those of a BM solution. Consequently, when the EYMH coupling constant $\alpha=\sqrt{4 \pi G} v$ (where $v$ is the Higgs field expectation value) is varied, the gravitating MAP solution starts from the flat space solution, reaches a critical solution at a maximal value of $\alpha$, and then with decreasing $\alpha$ smoothly reaches the BM solution in the limit $\alpha \rightarrow 0$ (after rescaling of the radial coordinate) [7. Consequently, we do not find a SAP solution in EYM theory, and similarly, we do not find new solutions, when $m=2 k=2, n>1$, since their boundary conditions agree with those of the axially symmetric solutions of 4 .

When $m=2 k=4$, however, the boundary conditions eq. (9) for the gauge field differ from those of the known solutions [1, 4. We therefore may expect the existence of new EYM solutions, subject to these boundary conditions. Indeed, for $m=4, n=4$, for instance, when $\alpha$ is varied again, the gravitating EYMH vortex solution starts from the flat space solution [8], reaches a critical solution at a maximal value of $\alpha$, and then with decreasing $\alpha$ smoothly reaches a new EYM solution in the limit $\alpha \rightarrow 0$ (after rescaling of the radial coordinate) [9].

Let us in the following refer to the solutions characterized by the integers $k$ and $n$ as $(k, n)$ solutions. In Fig. 1a we exhibit the energy density of the new $(2,4)$ EYM solution. Its energy density has a pronounced maximum on the $\rho$-axis, thus the energy density is torus-like. The energy density of this $(2,4)$ solution is thus similar to the energy density of the (lowest mass) known $(1,4)$ EYM solution [4, exhibited in Fig. 1b. But the maximum of 
the $(2,4)$ solution is smaller than the maximum of the $(1,4)$ solution, and it is shifted slightly inwards. In addition, the $(2,4)$ solution has a small saddle point on the $\rho$-axis, located further outwards. On the other hand comparison with the corresponding EYMH solution [8] shows, that the energy density of the EYM solution differs significantly from the energy density of the flat space $(2,4)$ EYMH solution, whose energy density exhibits two tori, located symmetrically with respect to the $x y$-plane 8 .

$\varepsilon \quad k=2, n=4$, lower branch

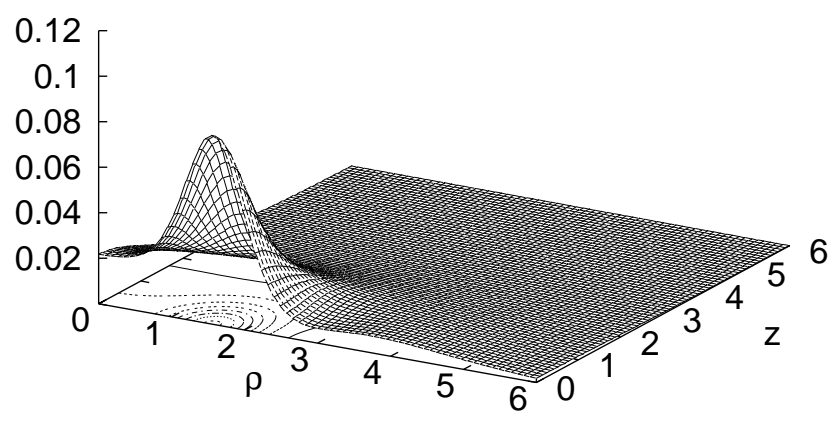

(a)

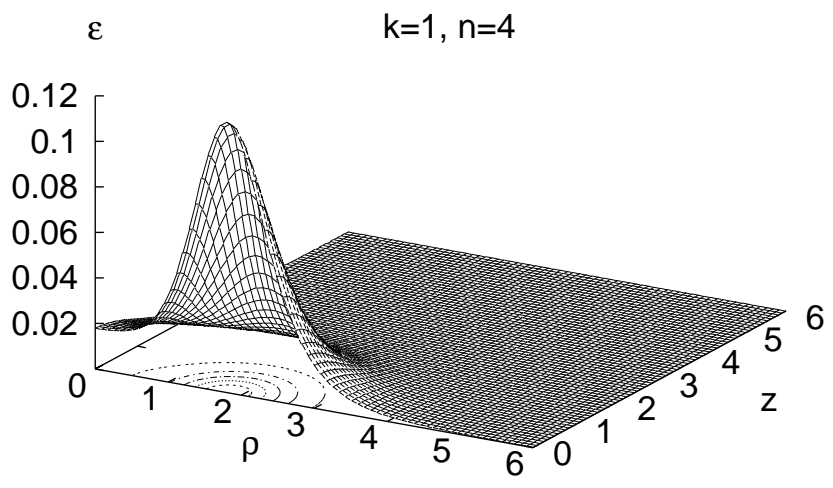

(b)

Fig. 1 The energy density of the $(2,4)$ EYM solution on the lower mass branch (a) and of the $(1,4)$ EYM solution (b).

The existence of a $k=2$ EYM solution with winding number $n=4$, immediately suggests that analogous solutions with winding number $n \neq 4$ 
should exist as well. Indeed, by increasing $n$, a whole set of such new solutions is obtained numerically, one for each integer $n$. On the other hand, a decrease of $n$ does not lead to new solutions. Instead a bifurcation at the non-integer and thus unphysical value of $n=3.7175$ is obtained, where a second branch of solutions with higher mass appears. Consequently there is a second solution for $n=4$, and also for each higher integer $n$, but there are no such solutions for integer $n \leq 3$. This is illustrated in Fig. 2a, where we exhibit the mass of these $(2, n)$ EYM solutions. Only the integer values of $n$ in the figure correspond to physical solutions. In Fig. $2 \mathrm{~b}$ we illustrate the value of the metric function $f$ at the origin. We note, that for $(1, n)$ solutions (with a single node) we find only one branch, which is also exhibited in Figs. 2.

(a)

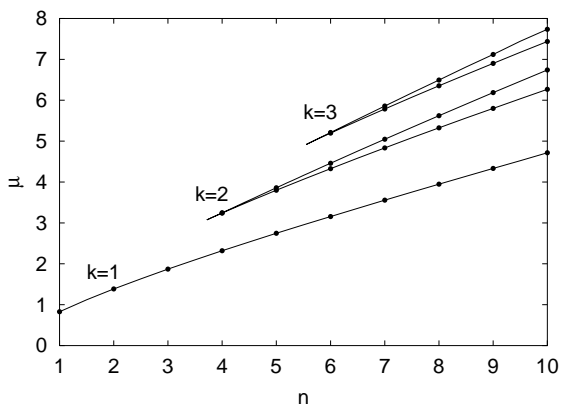

(b)

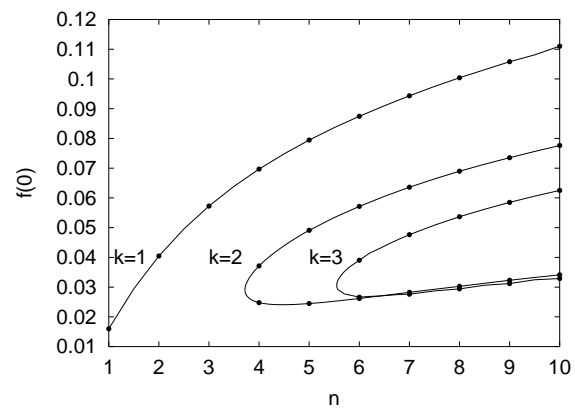

Fig. 2 The mass (a) and the value of the metric function $f$ at the origin (b) of the $(1, n),(2, n)$ and $(3, n)$ EYM solutions is shown as a function of $n$.

To illustrate the dependence of the solutions on $n$, we exhibit the energy density of the $(2, n)$ solutions with $n=4,6,8$ on the lower (mass) branch in Fig. 3a and on the upper branch in Fig. 3b. With increasing $n$ the maximum of the energy density decreases and moves outwards for the solutions on both branches. For the same $n$, the maximum of the upper mass solutions is higher and located further inwards than the maximum of the lower mass solutions. The small saddle point turns into a small maximum on the upper branch, thus the energy density of the solutions on the upper branch has a more complicated structure as seen in Fig. 4, where a surface of constant energy density is shown for the $(2,4)$ solution. We exhibit the gauge field function $\mathrm{H}_{2}$ for the same set of solutions in Figs. 5. 
(a)

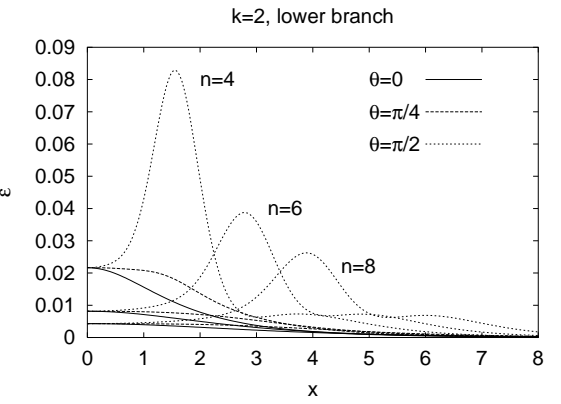

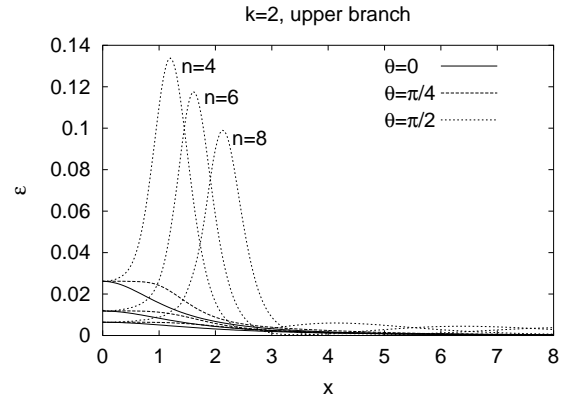

Fig. 3 The energy density of the $(2, n)$ EYM solution on the lower mass branch (a) and on the upper mass branch (b) for $n=4,6,8$.

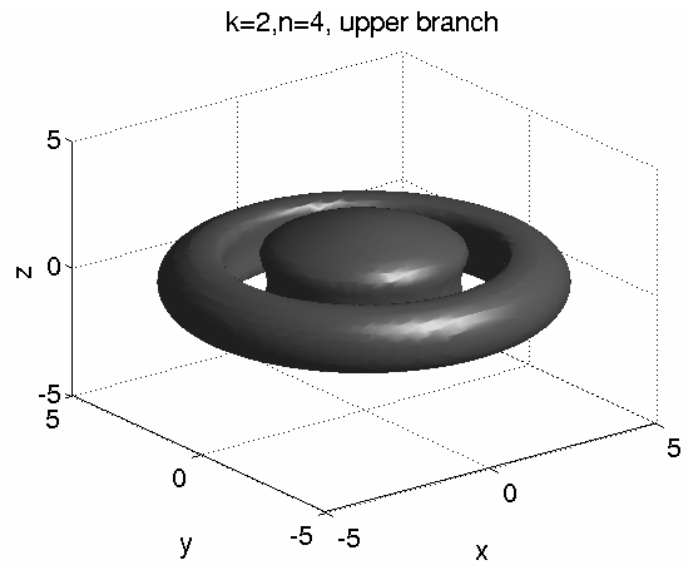

Fig. 4 A surface of constant energy density $(\varepsilon=0.005)$ of the $(2,4)$ EYM solution on the upper mass branch.

For $k=3$ we obtain a similar pattern of solutions. But the new type of solutions appears only for $n>5$. The mass and the value of the metric function $f$ at the origin of these $(3, n)$ solutions are also shown in Figs. 2. The energy density for $(3, n)$ solutions with $n=6,8,10$ and the gauge field function $\mathrm{H}_{2}$ are shown in Figs. 6 and 7, respectively. Based on these results, we conjecture the existence of $(k, n)$ solutions with $k>3$, but expect that they will appear only for still larger values of $n$. 
(a)

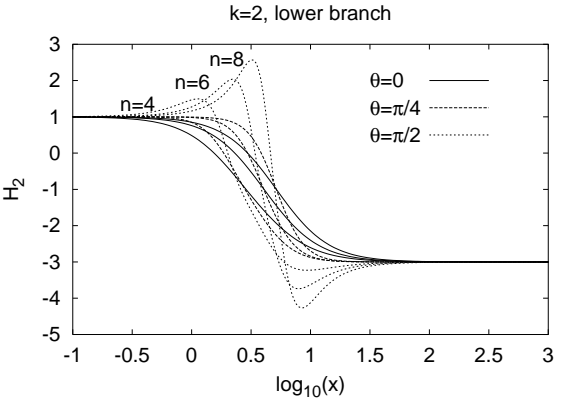

(b)

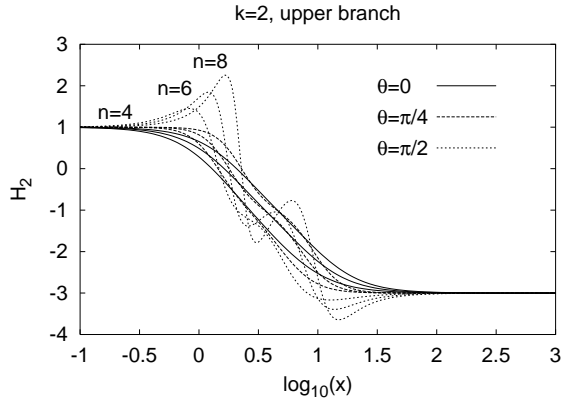

Fig. 5 The gauge field function $H_{2}$ of the $(2, n)$ EYM solutions on the lower mass branch (a) and on the upper mass branch (b) for $n=4,6,8$.

(a)

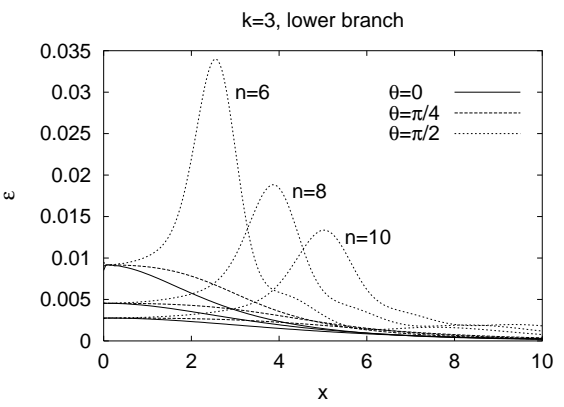

(b)

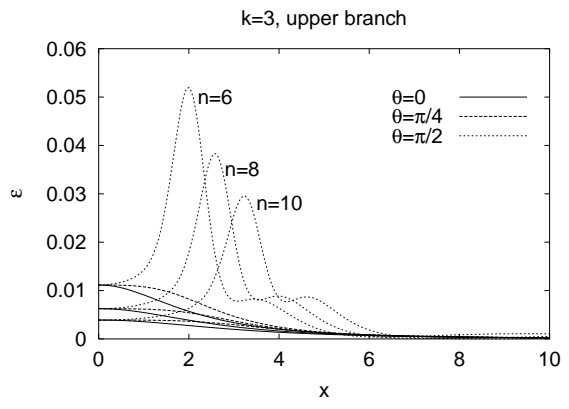

Fig. 6 The energy density of the $(3, n)$ EYM solution on the lower mass branch (a) and on the upper mass branch (b) for $n=6,8,10$.

(a)

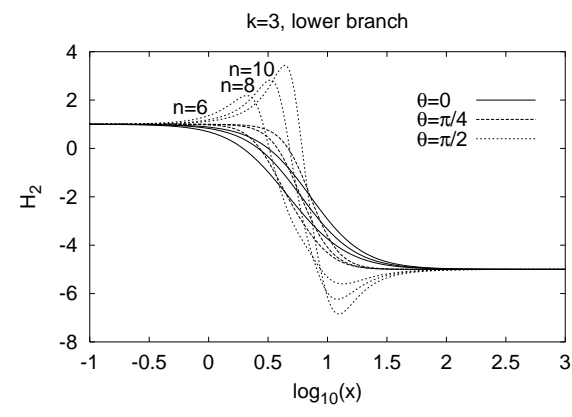

(b)

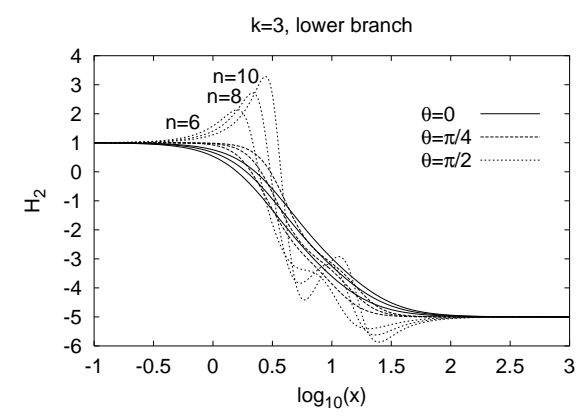

Fig. 7 The gauge field function $H_{2}$ of the $(3, n)$ EYM solutions on the lower mass branch (a) and on the upper mass branch (b) for $n=6,8,10$. 


\section{Conclusions}

We have constructed numerically new static regular solutions of EYM theory with axial symmetry. These solutions are characterized by the integers $(k, n)$, related to the azimuthal and polar angles, respectively. In particular, we have obtained solutions for $k=2, n=4-10$, and $k=3, n=6-10$.

Like the previously known $(1, n)$ solutions [4, the $(2, n)$ and $(3, n)$ solutions on the lower branch have a torus-like shape. On the upper branch of the $(3, n)$ solutions double tori appear for larger $n$. The $(1, n)$ solutions (with one node) most likely exist for any integer $n \geq 1$ and form a single branch. The new $(2, n)$ and $(3, n)$ solutions have lower bounds on $n, n=2 k$, which imply the existence of two branches of solutions for both values of $k$. We expect, that the $(2, n)$ and $(3, n)$ solutions represent only the first sequences of new solutions, and conjecture the existence of $(k, n)$ solutions also for higher values of $k$.

The $k=1 \mathrm{BM}$ solutions are unstable [3], and there is all reason to believe, that the new $(k, n)$ solutions are also unstable. Having constructed the regular axially symmetric solutions it appears straightforward to construct analogous black hole solutions, and, in particular, to look for rotating solutions 12 .

Acknowledgement

R.I. gratefully acknowledges support by the DAAD, and B.K. support by the DFG.

\section{References}

[1] R. Bartnik and J. McKinnon, Phys. Rev. Lett. 61 (1988) 141.

[2] M. S. Volkov and D. V. Galt'sov, Sov. J. Nucl. Phys. 51 (1990) 747;

P. Bizon, Phys. Rev. Lett. 64 (1990) 2844;

H. P. Künzle and A. K. M. Masoud-ul-Alam, J. Math. Phys. 31 (1990) 928.

[3] N. Straumann and Z. H. Zhou, Phys. Lett. B237 (1990) 353; B243 (1990) 33;

M. S. Volkov and D. V. Gal'tsov, Phys. Lett. B341 (1995) 279;

G. Lavrelashvili, and D. Maison, Phys. Lett. B343 (1995) 214; 
M. S. Volkov, O. Brodbeck, G. Lavrelashvili and N. Straumann, Phys. Lett. B349 (1995) 438.

[4] B. Kleihaus and J. Kunz, Phys. Rev. Lett. 78 (1997) 2527; Phys. Rev. Lett. 79 (1997) 1595; Phys. Rev. D57 (1998) 834; Phys. Rev. D57 (1998) 6138.

[5] K. Lee, V.P. Nair and E.J. Weinberg, Phys. Rev. D45 (1992) 2751. P. Breitenlohner, P. Forgacs and D. Maison, Nucl. Phys. B383 (1992) 357; B442 (1995) 126.

[6] B. Hartmann, B. Kleihaus, and J. Kunz, Phys. Rev. Lett. 86 (2001) 1422; Phys. Rev. D65 (2002) 024027.

[7] B. Kleihaus and J. Kunz, Phys. Rev. Lett. 85 (2000) 2430.

[8] B. Kleihaus, J. Kunz and Ya. Shnir, Phys. Lett. B570 (2003) 237; Phys. Rev. D68 (2003) 101701; Phys. Rev. D70 (2004) 065010.

[9] B. Kleihaus, J. Kunz, and Y. Shnir, in preparation.

[10] W. Schönauer and R. Weiß, J. Comput. Appl. Math. 27, 279 (1989) 279; M. Schauder, R. Weiß and W. Schönauer, The CADSOL Program Package, Universität Karlsruhe, Interner Bericht Nr. 46/92 (1992).

[11] Y. Brihaye, and J. Kunz, Phys. Rev. D50 (1994) 4175;

D. V. Gal'tsov and M. S. Volkov, Phys. Lett. B263 (1991) 255.

[12] B. Kleihaus and J. Kunz, Phys. Rev. Lett. 86 (2001) 3704; B. Kleihaus, J. Kunz, and F. Navarro-Lérida, Phys. Rev. D66 (2002) 104001; Phys. Rev. Lett. 90 (2003) 171101; Phys. Rev. D69 (2004) 064028; Phys. Lett. B599 (2004) 294. 